\title{
Effect of Statins, Metformin, Angiotensin-Converting Enzyme Inhibitors, and Angiotensin II Receptor Blockers on Age-Related Macular Degeneration
}

\author{
Hyesung Lee ${ }^{1}$, Ha-Lim Jeon ${ }^{1}$, Sang Jun Park ${ }^{2}$, and Ju-Young Shin ${ }^{1}$ \\ ${ }^{1}$ School of Pharmacy, Sungkyunkwan University, Suwon; \\ ${ }^{2}$ Department of Ophthalmology, Seoul National University Bundang Hospital, Seoul National University College of Medicine, Seongnam, Korea.
}

\begin{abstract}
Purpose: Statins, metformin, angiotensin-converting enzyme (ACE) inhibitors, and angiotensin receptor blockers (ARBs) have been suggested for treating age-related macular degeneration (AMD) due to their pleiotropic effects. Therefore, we investigated whether these drugs prevent AMD.

Materials and Methods: We conducted a nested case-control study using the Korean National Health Insurance Service database. Using risk-set sampling of age, sex, cohort entry date, and follow-up duration, we identified incident patients with AMD and 10 matching controls in cohorts with diabetes mellitus or cardiovascular diseases. Exposure was assessed within one year before the index date using patient prescription records. We conducted conditional logistic regression to estimate the odds ratios (ORs) and $95 \%$ confidence intervals (CIs) to evaluate the association between cardiovascular medications and AMD.

Results: Our study included 2330 cases and 23278 controls from a cohort of 231274 patients. The ORs (95\% CI) for AMD occurrence in users prescribed with statins, metformin, ACE inhibitors, and ARBs were 1.12 (0.94-1.32), 1.15 (0.91-1.45), 0.90 (0.611.34), and 1.21 (1.05-1.39), respectively. A duration-response was not observed.

Conclusion: Statins, metformin, ACE inhibitors, and ARBs did not inhibit AMD in elderly patients. The absence of a duration-response supports the lack of a causal relationship.
\end{abstract}

Key Words: Age-related macular degeneration, preventive effect, cardiovascular medications

\section{INTRODUCTION}

Age-related macular degeneration (AMD) is a progressive disease of the macula that affects central vision. In industrialized countries, it is the leading cause of irreversible blindness in people aged 65 years or older. ${ }^{1}$ The prevalence of AMD is likely to increase due to the exponential increase in aging populations. ${ }^{2}$ The etiology of AMD is not clearly understood; however,

Received: January 22, 2019 Revised: April 25, 2019

Accepted: May 19, 2019

Corresponding author: Ju-Young Shin, PhD, School of Pharmacy, Sungkyunkwan University, 2066 Seobu-ro, Jangan-gu, Suwon 16419, Korea.

Tel: 82-31-290-7702, Fax: 82-31-292-8800, E-mail: shin.jy@skku.edu

-The authors have no potential conflicts of interest to disclose.

(C) Copyright: Yonsei University College of Medicine 2019

This is an Open Access article distributed under the terms of the Creative Commons Attribution Non-Commercial License (https://creativecommons.org/licenses/ by-nc/4.0) which permits unrestricted non-commercial use, distribution, and reproduction in any medium, provided the original work is properly cited. several studies have suggested that immunologic and inflammatory processes ${ }^{3,4}$ and atherosclerosis ${ }^{5}$ are involved in its pathogenesis. In contrast, dietary intake of antioxidants has been associated with a decreased risk of exudative AMD. ${ }^{6}$ In Korea, the prevalence and incidence of wet AMD in a subset of the population aged 40 years or older were 36.43 (per 10000 people) and 3.02 (per 10000 person-years). ${ }^{7}$

Statins, ${ }^{8}$ metformin, ${ }^{9}$ angiotensin-converting enzyme (ACE) inhibitors, ${ }^{10}$ and angiotensin II receptor blockers (ARBs) ${ }^{11}$ are well-known medications that exert pleiotropic effects, such as anti-inflammatory and antioxidant effects, endothelium-protective actions, antiaggregatory and profibrinolytic effects, and atherosclerosis attenuation. Observational studies have examined the association of AMD with statins and ACE inhibitors. A systematic review reported that statin users experienced no preventive effect for AMD compared to non-users [relative risk $(\mathrm{RR})=0.95,95 \%$ confidence interval $(\mathrm{CI})=0.74-1.15] .{ }^{12} \mathrm{Ad}-$ ditionally, a nested case-control study found no decrease in 
the risk of AMD associated with ACE inhibitor use [odds ratio $(\mathrm{OR})=1.19,95 \% \mathrm{CI}=1.07-1.33]$ compared with non-use. ${ }^{13}$ Thus far, no previous studies have examined the association of metformin and ARBs with AMD incidence.

Using a cohort of elderly patients from the Korean National Health Insurance Service database, this nested case-control study aimed to determine whether users of statins, metformin, ACE inhibitors, or ARBs had a reduced risk of AMD compared to that in non-users.

\section{MATERIALS AND METHODS}

\section{Data sources}

We used the National Health Insurance Service-National Elderly Cohort (NHIS-NEC) database from South Korea. South Korea introduced the National Health Insurance program in 1977, and gradually expanded its coverage until it achieved universal healthcare in $1989 .{ }^{14} \mathrm{NHIS}$ collects the healthcare utilization records of the entire Korean population through claim data, which is generated by medical institutions for the reimbursement of costs covered by national insurance. ${ }^{15}$ Using the claim data, NHIS established a sample cohort database of elderly patients aged $\geq 60$ years. Through simple random sampling, NHIS-NEC consists of $10 \%$ of the total population aged $\geq 60$ years and eligible for health insurance or medical aid. ${ }^{16}$ This database includes demographic information and details on the healthcare services provided. Personal identification numbers were anonymized before providing information to researchers, and all diagnoses were coded according to the International Classification of Diseases, Tenth Revision (ICD-10).

\section{Cohort construction}

NHIS-NEC database was established from claim data from 2002-2015. Our study cohort included patients aged 65 years or older who were diagnosed with diabetes mellitus, including types 1 and 2 (ICD-10: E10-E14), or cardiovascular diseases (ICD-10: I05-I15, I20-I28, I30-I52, I60-I89) in 2002. This population was considered to contain patients with the aforementioned diseases, and cohort entry date was defined as the first date of diagnosis.

\section{Case definition and control selection}

We identified all incident patients with AMD between January 2012 and December 2015. Incident patients were defined as patients without a diagnostic history of degeneration of the macula and posterior pole (ICD-10: H35.3) before 2012, as diagnosis related to the disease of interest was specified by four digits in ICD code, which were introduced in 2011. Therefore, we excluded the patients who were diagnosed in 2011 or earlier from the study cohort. Cases were defined as all patients with incident AMD diagnosed with non-exudative (ICD-10: H35.30) or exudative (ICD-10: H35.31) AMD after the cohort entry date. To differentiate the cases from other cases with diseases related to the degeneration of the macula and posterior pole (ICD-10: H35.32-H35.39), we defined cases as patients with non-exudative or exudative AMD and not as those with other disease types. The first date of AMD diagnosis was defined as the index date. For each case, 10 controls were randomly selected by risk set sampling, and the controls were matched for sex, age, cohort entry date ( \pm 60 days), and follow-up duration.

\section{Exposure definition}

Statins, metformin, ACE inhibitors, and ARBs were the main exposures in this study. Therefore, we collected patient medication history until one year prior to index data collection. We defined exposure as a prescription history for single or combination administration of the aforementioned medications. Patients who were prescribed more than two types of these medications were defined as combination users, whereas the remaining patients were considered non-users. For subgroup analysis, we classified exposure more specifically according to the cumulative duration and timing of medication use. Cumulative duration was calculated as total days of medication prescription without duplicated days, and it was classified into three categories as follows: 1) fewer than 90 days, 2) fewer than 300 days, and 3) greater than 300 days. Additionally, the timing of medication use was classified into three categories as follows: 1) recent use, 2) current use, and 3) past use. We defined recent use as when the latest administration duration of the study medications overlapped with the index date. Current use was defined as cases in which the latest administration duration of the study medications occurred within 30 days from the index date, whereas the remaining cases were defined as past use.

\section{Potential confounders}

The following baseline characteristics and clinical details for all of the study subjects were considered possible confounders of the association between medication use and AMD: income level, health insurance type, Charlson comorbidity index, number of healthcare resources used, disease history, and medications. Disease history included cerebrovascular disease, complicated diabetes, uncomplicated diabetes, hyperlipidemia, hypertension, liver disease, myocardial infarction, and peripheral vascular disease. Medication history included the use of alpha-blockers, alpha-glucosidase, aspirin, beta-blockers, calcium channel blockers, diuretics, meglitinide, sulfonylurea, and thiazolidinedione. Claim data from over one year before the index date were used to define the number of healthcare resources used and disease and medication history.

\section{Statistical analysis}

We conducted a nested case-control study to investigate the preventive effect of statins, metformin, ACE inhibitors, ARBs, and their combinations on AMD. To compare the general char- 
acteristics between the case and control groups, we described categorical data with frequencies or proportions and continuous data with means and standard deviations. Furthermore, we conducted chi-square tests for unmatched categorical variables. To overcome the multiplicity problem for variables with more than two groups, we applied Bonferroni method. The level of significance was adjusted by $0.05 / \mathrm{k}$, where $\mathrm{k}$ was the number of groups for each variable. For primary analysis, we used conditional logistic regression to estimate the ORs and corresponding 95\% CIs for AMD in patients who used the study medications and their combinations versus non-users. These ORs assume unbiased estimates of the hazard ratios due to the use of risk set sampling. ${ }^{17}$ To adjust conditional logistic regression model, we used all of the potential confounders previously defined. For secondary analysis, we repeated the primary analysis, including cumulative duration and timing of medication use. To identify statistically significant differences in variables, including age, sex, AMD type, and several comorbidities, between cases and controls, we performed an interaction test and conducted subgroup analyses for variables with interaction effects. All statistical analyses were performed using SAS 9.4 for Windows (SAS Institute, Inc., Cary, NC, USA). The present study protocol was reviewed and approved by the Institutional Review Board of Sungkyunkwan University (approval no. 2018-02-008).

\section{RESULTS}

Of the 231274 patients diagnosed with diabetes mellitus or cardiovascular diseases in 2002, the study cohort included 200333 patients (Fig. 1), with 2330 incident cases and their 23278 matched controls. Matching ratio by risk set sampling was 1:9.99. There were several substantial differences in the baseline characteristics of subjects (Table 1). Cases were more likely to show greater healthcare resource use and higher Charlson comorbidity index and prevalence of comorbidities, including cerebrovascular diseases, complicated diabetes mellitus, uncomplicated diabetes mellitus, and peripheral vascular diseases. Moreover, compared with controls, cases were more likely to have a history of combined medication use, excluding aspirin, beta-blockers, and thiazolidinedione.

Compared to non-users, the adjusted ORs and their 95\% CIs for the occurrence of AMD in users of statins, metformin, ACE inhibitors, ARBs, and all medication combinations were 1.12 (0.94-1.32), 1.15 (0.91-1.45), 0.90 (0.61-1.34), 1.21 (1.05-1.39), and 1.21 (1.06-1.38), respectively (Table 2 ).

In secondary analyses, there were no significant associations of AMD prevention with the long-term use and timing of medication use for any of the medications evaluated (Tables 3 and 4). This finding was supported by the results of subgroup analyses for age group, sex, and peripheral vascular disease presence. Interaction test showed a significant difference in the results according to the type of AMD and presence of cerebrovascular diseases; however, the results of subgroup analyses were statistically insignificant (Fig. 2).

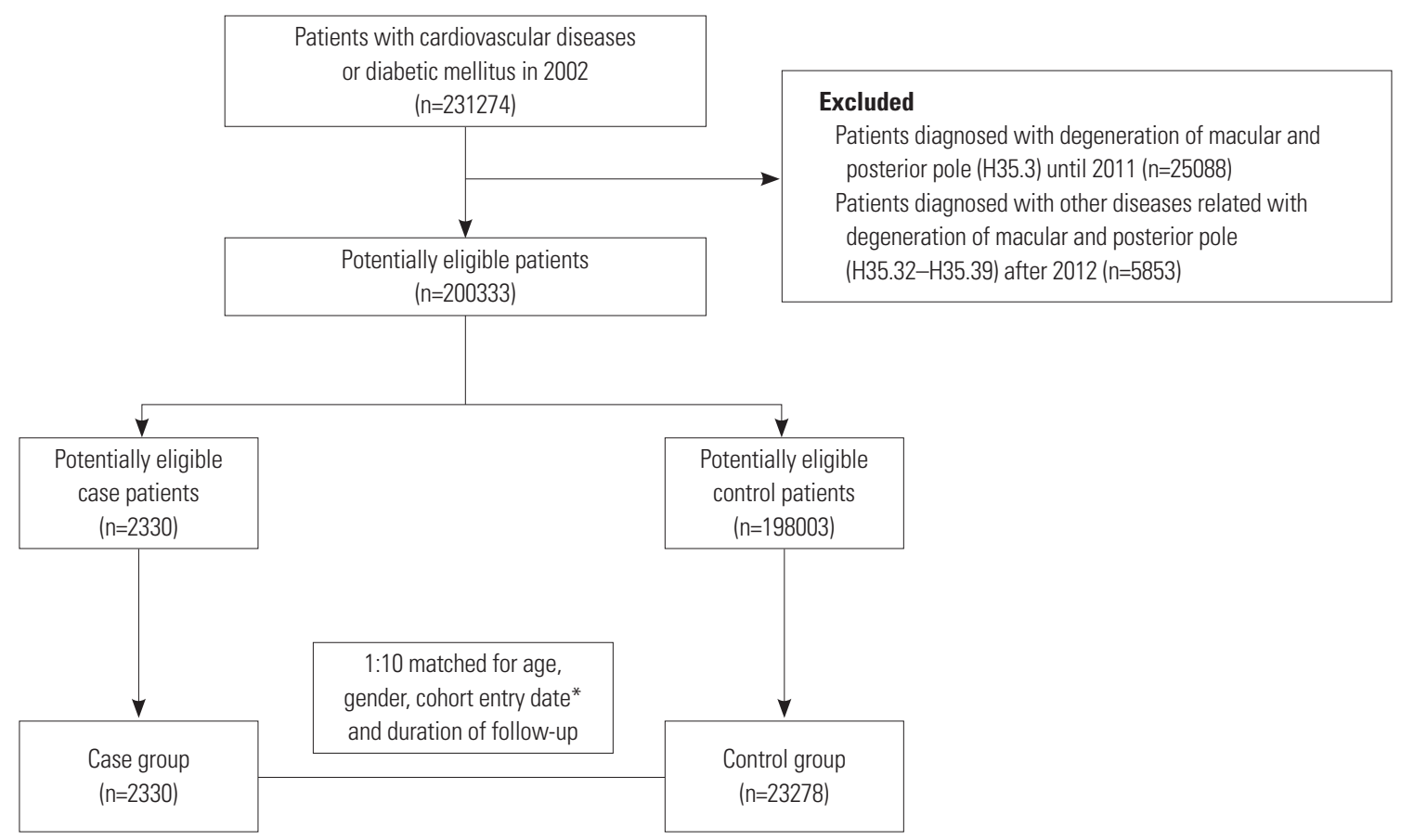

Fig. 1. Flowchart of study cohort construction. *Cohort entry date was matched with the cohort entry date of the cases, ranging from 30 days before or after the entry date. 
Table 1. Baseline Demographic and Clinical Characteristics of the Study Population

\begin{tabular}{|c|c|c|c|}
\hline Characteristics & $\begin{array}{l}\text { Cases }^{*} \\
(n=2330)\end{array}$ & $\begin{array}{l}\text { Controls* }^{*} \\
(\mathrm{n}=23278)\end{array}$ & $p$ value \\
\hline Age group & & & $\mathrm{N} / \mathrm{A}$ \\
\hline Mean $\pm S D(y r)$ & $66.5 \pm 5.0$ & $66.4 \pm 5.0$ & \\
\hline$<66$ & $1136(48.8)$ & $11360(48.8)$ & \\
\hline $66-70$ & $722(31.0)$ & $7220(31.0)$ & \\
\hline $71-75$ & $351(15.1)$ & $3510(15.1)$ & \\
\hline $76-80$ & $97(4.2)$ & $970(4.2)$ & \\
\hline $81-85$ & $18(0.8)$ & $180(0.8)$ & \\
\hline$>85$ & $6(0.3)$ & $38(0.2)$ & \\
\hline Sex & & & $\mathrm{N} / \mathrm{A}$ \\
\hline Female & $1471(63.1)$ & $14710(63.2)$ & \\
\hline Male & 859 (36.9) & $8568(36.8)$ & \\
\hline Income level $\left.\right|^{t, \ddagger}$ & & & 0.720 \\
\hline 00-02 & $363(15.6)$ & $3616(15.5)$ & \\
\hline 03-05 & $461(19.8)$ & $4651(20.0)$ & \\
\hline 06-08 & $707(30.3)$ & $7277(31.3)$ & \\
\hline $09-010$ & $799(34.3)$ & $7734(33.2)$ & \\
\hline Type of health insurance & & & 0.957 \\
\hline Health insurance & 2316 (99.4) & $23136(99.4)$ & \\
\hline Medical aid & $14(0.6)$ & $142(0.6)$ & \\
\hline No. healthcare resources used ${ }^{\dagger}$ & & & $<0.001$ \\
\hline Mean $\pm S D$ & $46.9 \pm 37.7$ & $37.6 \pm 34.9$ & \\
\hline$<16$ & $345(14.8)$ & $6624(28.5)$ & \\
\hline $16-24$ & $535(23.0)$ & $5726(24.6)$ & \\
\hline $25-40$ & $659(28.3)$ & $5511(23.7)$ & \\
\hline$>41$ & $791(33.9)$ & $5417(23.3)$ & \\
\hline \multicolumn{4}{|l|}{ Comorbidities } \\
\hline Cerebrovascular diseases & $941(40.4)$ & $8253(35.5)$ & $<0.001$ \\
\hline Complicated diabetes mellitus & $260(11.2)$ & $2210(9.5)$ & 0.009 \\
\hline Uncomplicated diabetes mellitus & $501(21.5)$ & $4324(18.6)$ & 0.001 \\
\hline Hyperlipidemia & $43(1.8)$ & $375(1.6)$ & 0.394 \\
\hline Hypertension & $0(0.0)$ & $3(0.0)$ & 1.000 \\
\hline Liver diseases & $32(1.4)$ & $325(1.4)$ & 0.929 \\
\hline Myocardial infarction & $32(1.4)$ & $257(1.1)$ & 0.241 \\
\hline Peripheral vascular diseases & $489(21.0)$ & $4245(18.2)$ & $<0.001$ \\
\hline \multicolumn{4}{|l|}{ Combined medications } \\
\hline Alpha-blockers & $987(42.4)$ & 9278 (39.9) & 0.019 \\
\hline Alpha-glucosidase & $536(23.0)$ & $4879(21.0)$ & 0.021 \\
\hline Aspirin & $25(1.1)$ & $197(0.8)$ & 0.261 \\
\hline Beta-blockers & $119(5.1)$ & $1094(4.7)$ & 0.377 \\
\hline Calcium channel blockers & $70(3.0)$ & $465(2.0)$ & 0.001 \\
\hline Diuretics & $1034(44.4)$ & $9739(41.8)$ & 0.018 \\
\hline Meglitinide & $1358(58.3)$ & $12676(54.5)$ & $<0.001$ \\
\hline Sulfonylurea & $743(31.9)$ & $6602(28.4)$ & $<0.001$ \\
\hline Thiazolidinedione & $138(5.9)$ & $1357(5.8)$ & 0.855 \\
\hline Charlson comorbidity index ${ }^{\dagger}$ & & & $<0.001$ \\
\hline Mean $\pm S D$ & $0.9 \pm 0.6$ & $0.8 \pm 0.6$ & \\
\hline
\end{tabular}

Table 1. Baseline Demographic and Clinical Characteristics of the Study Population (Continued)

\begin{tabular}{|c|c|c|c|}
\hline Characteristics & $\begin{array}{l}\text { Cases* }^{*} \\
(n=2330)\end{array}$ & $\begin{array}{l}\text { Controls* } \\
(\mathrm{n}=23278)\end{array}$ & $p$ value \\
\hline 0 & $681(29.2)$ & 7872 (33.8) & \\
\hline $1-2$ & $1315(56.4)$ & $12643(54.3)$ & \\
\hline$>3$ & 334 (14.3) & $2763(11.9)$ & \\
\hline
\end{tabular}

SD, standard deviation; N/A, not applicable.

Variables are presented as a number (percentage) unless otherwise noticed. *Cases and controls are matched by age, sex, cohort entry date, and follow-up duration; ${ }^{\dagger}$ According to Bonferroni method, the level of significance was adjusted by $0.05 / k$, where $k$ is the number of groups for each variable; " Income levels are classified into 11 groups ranging from 0-10, according to the type of health insurance. Ten of the groups are for employee and district subscribers. Group 0 indicates medical aid.

\section{DISCUSSION}

We found that statin, metformin, ACE inhibitor, or ARB use did not decrease the risk of AMD in an aged population after adjusting for several potential confounders, such as socioeconomic status, healthcare resource utilization, combined medication use, and comorbidities. No significant associations were observed between AMD risk and cumulative duration or timing of medication use. Additionally, there were no considerable differences between age groups, sex, AMD types, presence of cerebrovascular or peripheral vascular diseases, and uncomplicated or complicated diabetes mellitus.

Several studies have investigated the association between the risk of AMD and statins, and found no preventive effects. A case-control study suggested that statin use and AMD incidence have no association ( $\mathrm{OR}=0.93,95 \% \mathrm{CI}=0.81-1.07){ }^{18} \mathrm{In}$ a 5 -year cohort study, statins did not prevent early age-related maculopathy (early onset: $\mathrm{OR}=1.12,95 \% \mathrm{CI}=0.47-2.67$; progression: $\mathrm{OR}=1.22,95 \% \mathrm{CI}=0.54-2.76$; late incidence: $\mathrm{OR}=0.41$, 95\% $\mathrm{CI}=0.12-1.45) .{ }^{19}$ In contrast, a nested case-control study reported a risk for AMD with statin use $(\mathrm{OR}=1.30,95 \% \mathrm{CI}=1.17-$ 1.44).$^{13}$ However, the findings of other studies conflict with our results. According to one cross sectional study, ${ }^{20}$ the relationship between statin use and AMD was negative ( $O R=0.14,95 \%$ $\mathrm{CI}=0.02-0.83$ ). Moreover, several studies have demonstrated preventive effects of statins. ${ }^{21-23}$

Some studies have investigated the association between the risk of AMD and use of ACE inhibitors or ARBs. A nested casecontrol study reported that ACE inhibitor use may be associated with an increased risk of AMD (adjusted RR=1.19, 95\% $\mathrm{CI}=1.07-1.33) .{ }^{13} \mathrm{~A}$ study that analyzed data pooled from three population-based cohort studies found no significant relationship between ACE inhibitor use and the incidence of early age-related maculopathy in each of the three cohort studies, as well as in the pooled cohort (OR=1.1, 95\% $\mathrm{CI}=0.8-1.5){ }^{24} \mathrm{~A}$ casecontrol design study showed that ARBs have a protective effect on the development of choroidal neovascularization, which is a late form of AMD, in patients with AMD. ${ }^{25}$ However, these 
Hyesung Lee, et al.

Table 2. Association between Age-Related Macular Degeneration and Exposure

\begin{tabular}{lcccc}
\hline \multicolumn{1}{c}{ Exposure } & Cases $^{*}(\mathbf{n}=\mathbf{2 3 3 0})$ & Controls* $^{*}(\mathbf{n}=\mathbf{2 3 2 7 8 )}$ & Crude OR (95\% CI) & Adjusted ORs ${ }^{\dagger}$ (95\% CI) \\
\hline None & $551(23.7)$ & $6900(29.6)$ & 1.00 (Reference) & $1.00($ Reference) \\
Statins only & $210(9.0)$ & $2009(8.6)$ & $1.33(1.12-1.57)$ & $1.12(0.94-1.32)$ \\
Metformin only & $111(4.8)$ & $1062(4.6)$ & $1.33(1.07-1.64)$ & $1.15(0.91-1.45)$ \\
ACE inhibitors only & $28(1.2)$ & $367(1.6)$ & $0.97(0.65-1.43)$ & $0.90(0.61-1.34)$ \\
ARBs only & $456(19.6)$ & $4319(18.6)$ & $1.33(1.17-1.52)$ & $1.21(1.05-1.39)$ \\
All combinations & $974(41.8)$ & $8621(37.0)$ & $1.44(1.29-1.61)$ & $1.21(1.06-1.38)$ \\
\hline
\end{tabular}

OR, odds ratio; Cl, confidence interval; ACE inhibitors, angiotensin-converting enzyme inhibitors; ARBs, angiotensin II receptor blockers.

Variables are presented as a number (percentage) unless otherwise noticed.

${ }^{*}$ Cases and controls are matched by age, sex, cohort entry date, and follow-up duration; ${ }^{\dagger}$ Adjusted for income level, Charlson comorbidity index, the number of prescriptions, cerebrovascular disease history, complicated or uncomplicated diabetes, hyperlipidemia, hypertension and peripheral vascular disease, and the use of alpha-blockers, alpha-glucosidase, aspirin, beta-blockers, calcium channel blockers, diuretics, meglitinide, sulfonylurea, or thiazolidinedione.

Table 3. Association between Age-Related Macular Degeneration and Exposure according to Cumulative Duration

\begin{tabular}{|c|c|c|c|c|}
\hline Exposure & Cases $^{*}(n=2330)$ & Controls* $(n=23278)$ & Crude OR (95\% CI) & Adjusted ORs ${ }^{\dagger}(95 \% \mathrm{Cl})$ \\
\hline None & $551(23.7)$ & $6900(29.6)$ & 1.00 (Reference) & 1.00 (Reference) \\
\hline \multicolumn{5}{|l|}{ Statins only } \\
\hline$<90$ days & $33(1.4)$ & $268(1.2)$ & $1.56(1.07-2.26)$ & $1.30(0.90-1.90)$ \\
\hline 90-299 days & $77(3.3)$ & 877 (3.8) & $1.11(0.87-1.43)$ & $0.95(0.74-1.22)$ \\
\hline$\geq 300$ days & $100(4.3)$ & $864(3.7)$ & $1.47(1.17-1.84)$ & $1.22(0.97-1.53)$ \\
\hline \multicolumn{5}{|l|}{ Metformin only } \\
\hline$<90$ days & $14(0.6)$ & $109(0.5)$ & $1.62(0.92-2.85)$ & $1.35(0.76-2.39)$ \\
\hline 90-299 days & $45(1.9)$ & $333(1.4)$ & $1.71(1.24-2.36)$ & $1.50(1.07-2.09)$ \\
\hline$\geq 300$ days & $52(2.2)$ & $620(2.7)$ & $1.07(0.79-1.43)$ & $0.93(0.68-1.26)$ \\
\hline \multicolumn{5}{|c|}{ ACE inhibitors only } \\
\hline$<90$ days & $0(0.0)$ & $22(0.1)$ & $\mathrm{N} / \mathrm{A}$ & $\mathrm{N} / \mathrm{A}$ \\
\hline 90-299 days & $12(0.5)$ & $128(0.6)$ & $1.19(0.65-2.17)$ & $1.18(0.64-2.15)$ \\
\hline$\geq 300$ days & $16(0.7)$ & $217(0.9)$ & $0.94(0.56-1.57)$ & $0.87(0.52-1.45)$ \\
\hline \multicolumn{5}{|l|}{ ARBs only } \\
\hline$<90$ days & $39(1.7)$ & $384(1.7)$ & $1.27(0.90-1.78)$ & $1.07(0.76-1.52)$ \\
\hline 90-299 days & $158(6.8)$ & 1378 (5.9) & $1.45(1.20-1.74)$ & $1.35(1.11-1.64)$ \\
\hline$\geq 300$ days & $259(11.1)$ & $2557(11.0)$ & $1.28(1.10-1.50)$ & $1.16(0.98-1.36)$ \\
\hline \multicolumn{5}{|l|}{ All combinations } \\
\hline$<90$ days & $19(0.8)$ & $166(0.7)$ & $1.44(0.89-2.33)$ & $1.31(0.80-2.13)$ \\
\hline 90-299 days & $249(10.7)$ & 2274 (9.8) & 1.39 (1.19-1.63) & $1.23(1.04-1.47)$ \\
\hline$\geq 300$ days & 706 (30.3) & $6181(26.6)$ & $1.46(1.29-1.64)$ & $1.20(1.05-1.38)$ \\
\hline
\end{tabular}

OR, odds ratio; Cl, confidence interval; ACE inhibitors, angiotensin-converting enzyme inhibitors; ARBs, angiotensin II receptor blockers; N/A, not applicable. Variables are presented as a number (percentage) unless otherwise noticed.

${ }^{*}$ Cases and controls are matched by age, sex, cohort entry date, and follow-up duration; ${ }^{\dagger}$ Adjusted for income level, Charlson comorbidity index, the number of prescriptions, cerebrovascular disease history, complicated or uncomplicated diabetes, hyperlipidemia, hypertension and peripheral vascular disease, and the use of alpha-blockers, alpha-glucosidase, aspirin, beta-blockers, calcium channel blockers, diuretics, meglitinide, sulfonylurea, or thiazolidinedione.

authors concluded that ARBs were unlikely to confer a preventive effect against choroidal neovascularization in patients with AMD.

We examined whether these medications protected against AMD using a nested case-control study design. This observational study design integrates the concept of traditional casecontrol studies within a constructed cohort. ${ }^{26}$ Moreover, this study design overcomes some of the disadvantages associated with case-control studies, while incorporating the advantages of cohort studies. Particularly, selection bias, which is a consid- erable concern in traditional case-control studies, is minimized by selecting cases and controls from populations with the same risk. Moreover, information bias is decreased. However, this study design had some disadvantages, including the fact that all pertinent risk factors were not recorded and the statistical power was low due to the small sample sizes.

Our study had several strengths. First, we used NHIS-NEC data sourced from a well-established and validated national longitudinal database, and included follow-up data from 20022015 for 558147 elderly subjects. Additionally, this database was 
Table 4. Association between Age-Related Macular Degeneration and Exposure according to the Time of Administration

\begin{tabular}{|c|c|c|c|c|}
\hline Exposure & Cases $^{*}(n=2330)$ & Controls* $(n=23278)$ & Crude OR (95\% CI) & Adjusted $\mathrm{ORs}^{\dagger}(95 \% \mathrm{Cl})$ \\
\hline None & $551(23.7)$ & $6900(29.6)$ & 1.00 (Reference) & 1.00 (Reference) \\
\hline \multicolumn{5}{|l|}{ Statins only } \\
\hline Current use & $164(7.0)$ & $1527(6.6)$ & $1.37(1.14-1.64)$ & $1.13(0.94-1.37)$ \\
\hline Recent use & $16(0.7)$ & $145(0.6)$ & $1.41(0.84-2.39$ & $1.28(0.76-2.17)$ \\
\hline Past use & $30(1.3)$ & $337(1.5)$ & $1.13(0.77-1.65)$ & $0.97(0.66-1.42)$ \\
\hline \multicolumn{5}{|l|}{ Metformin only } \\
\hline Current use & $81(3.5)$ & $846(3.6)$ & $1.22(0.96-1.56)$ & $1.07(0.82-1.39)$ \\
\hline Recent use & $11(0.5)$ & $99(0.4)$ & $1.42(0.76-2.66)$ & $1.25(0.66-2.36)$ \\
\hline Past use & $19(0.8)$ & $117(0.5)$ & $2.05(1.25-3.35)$ & $1.65(1.00-2.73)$ \\
\hline \multicolumn{5}{|c|}{ ACE inhibitors only } \\
\hline Current use & $25(1.1)$ & $311(1.3)$ & $1.02(0.67-1.54)$ & $0.96(0.63-1.46)$ \\
\hline Recent use & $3(0.1)$ & $25(0.1)$ & $1.54(0.46-5.10)$ & $1.45(0.43-4.89)$ \\
\hline Past use & $0(0.0)$ & $31(0.1)$ & $\mathrm{N} / \mathrm{A}$ & $\mathrm{N} / \mathrm{A}$ \\
\hline \multicolumn{5}{|l|}{ ARBs only } \\
\hline Current use & $382(16.4)$ & $3526(15.2)$ & $1.37(1.20-1.57)$ & $1.25(1.08-1.45)$ \\
\hline Recent use & $34(1.5)$ & $318(1.4)$ & $1.34(0.93-1.94)$ & $1.22(0.84-1.77)$ \\
\hline Past use & $40(1.7)$ & $475(2.0)$ & $1.05(0.75-1.47)$ & $0.91(0.65-1.28)$ \\
\hline \multicolumn{5}{|l|}{ All combinations } \\
\hline Current use & $906(38.9)$ & 7803 (33.5) & $1.48(1.32-1.66)$ & $1.25(1.09-1.42)$ \\
\hline Recent use & $47(2.0)$ & $470(2.0)$ & $1.28(0.93-1.74)$ & $1.09(0.79-1.50)$ \\
\hline Past use & $21(0.9)$ & $348(1.5)$ & $0.76(0.49-1.20)$ & $0.69(0.44-1.09)$ \\
\hline
\end{tabular}

OR, odds ratio; $\mathrm{Cl}$, confidence interval; ACE inhibitors, angiotensin-converting enzyme inhibitors; ARBs, angiotensin II receptor blockers, N/A, not applicable. Variables are presented as a number (percentage) unless otherwise noticed.

${ }^{*}$ Cases and controls are matched by age, sex, cohort entry date, and follow-up duration; ${ }^{\dagger}$ Adjusted for income level, Charlson comorbidity index, the number of prescriptions, cerebrovascular disease history, complicated or uncomplicated diabetes, hyperlipidemia, hypertension and peripheral vascular disease, and the use of alpha-blockers, alpha-glucosidase, aspirin, beta-blockers, calcium channel blockers, diuretics, meglitinide, sulfonylurea, or thiazolidinedione.

constructed by collecting reimbursement data from all inpatient and outpatient claim records under a fee-for-service system, enabling researchers to collect detailed information about the utilization of healthcare resources. Second, the results of this study have high external validity and generalizability, since the database we used included data from the entire population of South Korea. Therefore, our findings reflect the realworld situation.

There were some limitations to this study. Although the claim database we used contained a large sample that was representative of the population, there are concerns regarding its validity. AMD cases were defined according to the diagnoses listed in the database, which may differ from the actual diagnoses received by the patients. Although the overall positive predictive value of diagnosis was $70 \%,{ }^{27}$ validity remains an issue. Additionally, information on medication use was based solely on prescriptions, and the actual administration may have differed. Furthermore, we could not include variables related to lifestyle, family history, and cardiovascular factors (e.g., blood pressure, $\mathrm{HbAlc}$, and lipid profile), which are possible risk factors of $\mathrm{AMD}$, as this information was absent from the database. However, confounding by cardiovascular factors would be limited since our study design included a cohort of patients with cardiovascular diseases or diabetes mellitus and adjustments for comorbidities relevant to cardiovascular disorders. Moreover, there is a possibility of selection bias in which actual patients with AMD were misclassified as controls, since we could not confirm the use of optical coherence tomography, which was not a reimbursed procedure during the study period, for the diagnosis of AMD. However, this would not cause substantial bias in our results, since there is no conclusive evidence for differential disease misclassification rates between exposed and unexposed patients. Finally, a previous study showed that statins have protective effects against early-stage and exudative AMD. However, we could not conduct a subgroup analysis for AMD stage as specific stages could not be identified using our database. Moreover, in the database, there was no information about the number of treated eyes. Therefore, the intracorrelation between eyes could not be investigated. This limitation may have masked any preventive effects of the medications.

In conclusion, we found no evidence that statins, metformin, ACE inhibitors, or ARBs prevented the occurrence of AMD in elderly patients. We also found no significant relationships between the risk of AMD and duration-response or timing of medication use. Moreover, we found no significant differences between age groups, sex, AMD types, and presence of cerebrovascular diseases, complicated or uncomplicated diabetes mellitus, or peripheral vascular diseases. 


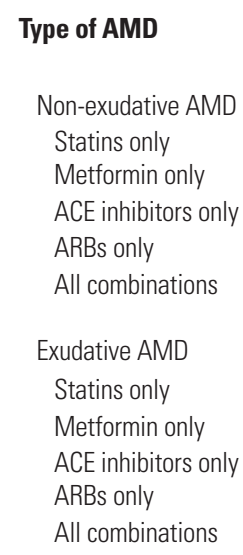

All combinations

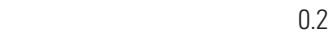

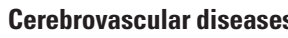 \\ No

$$
\begin{aligned}
& \text { Statins only } \\
& \text { Metformin only } \\
& \text { ACE inhibitors only } \\
& \text { ARBs only } \\
& \text { All combinations }
\end{aligned}
$$

Yes

Statins only

Metformin only

ACE inhibitors only

ARBs only

All combinations

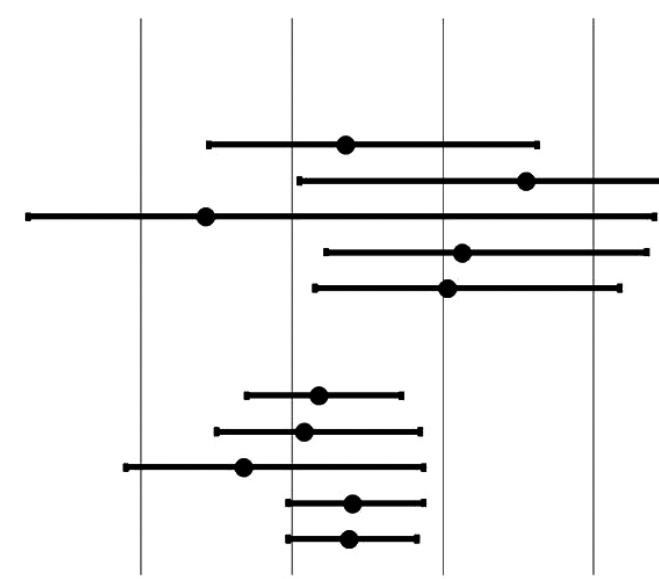

20.6

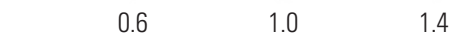

.4

1.8
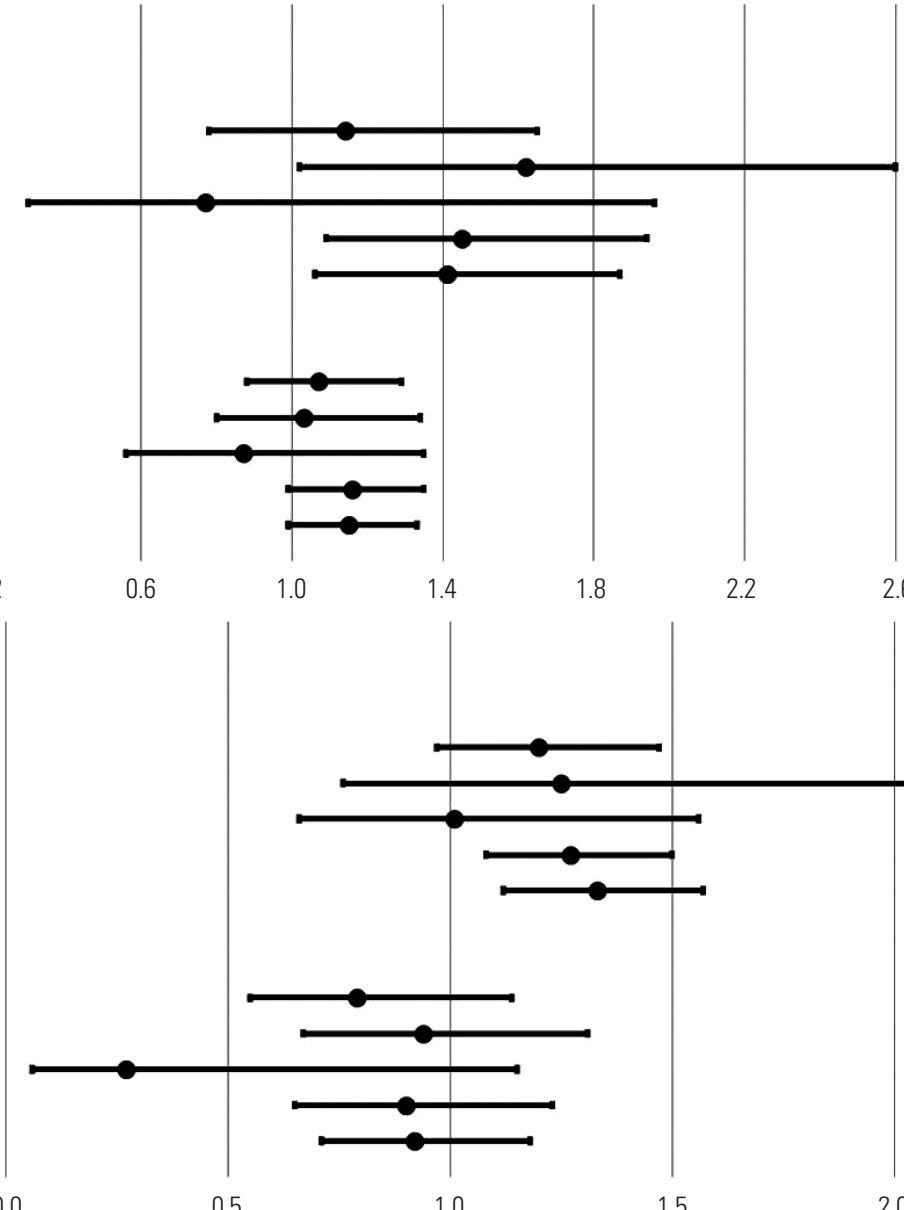

aORs $(95 \% \mathrm{CI}) *$

$1.14(0.78-1.65)$

$1.62(1.02-2.60)$

$0.77(0.30-1.96)$

$1.45(1.09-1.94)$

$1.41(1.06-1.87)$

$1.07(0.88-1.29)$

$1.03(0.80-1.34)$

$0.87(0.56-1.35)$

$1.16(0.99-1.35)$

$1.15(0.99-1.33)$

0.0

0.5

1.0

2.6

aORs $(95 \% \mathrm{CI})^{*}$

$1.20(0.97-1.47)$

$1.25(0.76-2.05)$

1.01 (0.66-1.56)

$1.27(1.08-1.50)$

$1.33(1.12-1.57)$

$0.79(0.55-1.14)$

$0.94(0.67-1.31)$

0.27 (0.06-1.15)

$0.90(0.65-1.23)$

0.92 (0.71-1.18)

Fig. 2. Subgroup analyses of the associations between AMD and exposures according to the type of AMD and presence of cerebrovascular diseases. *Adjusted for income level, Charlson comorbidity index, the number of prescriptions, cerebrovascular disease history, complicated or uncomplicated diabetes, hyperlipidemia, hypertension and peripheral vascular disease, and the use of alpha-blockers, alpha-glucosidase, aspirin, beta-blockers, calcium channel blockers, diuretics, meglitinide, sulfonylurea, or thiazolidinedione. AMD, age-related macular degeneration; ACE inhibitors, angiotensin-converting enzyme inhibitors; ARBs, angiotensin II receptor blockers; aORs, adjusted odds ratios; $\mathrm{Cl}$, confidence interval.

\section{ACKNOWLEDGEMENTS}

National Health Insurance Service (NHIS) provided access to the NHIS-National Elderly Cohort database. The authors would like to thank NHIS for its cooperation.

This study was supported by a grant from the Basic Science Research Program of the National Research Foundation of Korea (NRF), which was founded by the Ministry of Education (Grant number: NRF-2017R1C1B5017907).

\section{AUTHOR CONTRIBUTIONS}

Conceptualization: Ju-Young Shin. Data curation: Hyesung Lee. Formal analysis: Hyesung Lee. Funding acquisition: Ju-Young Shin. Investigation: Hyesung Lee. Methodology: Ju-Young Shin and Hyesung Lee. Project administration: Ju-Young Shin. Resources: Ha-Lim Jeon. Software: Hyesung Lee. Supervision: Ju-Young Shin. Validation: JuYoung Shin and Ha-Lim Jeon. Visualization: Hyesung Lee. Writingoriginal draft: Hyesung Lee and Ha-Lim Jeon. Writing—review \& editing: Ju-Young Shin and Sang Jun Park.

\section{ORCID iDs}

Hyesung Lee Ha-Lim Jeon Sang Jun Park Ju-Young Shin https://orcid.org/0000-0001-6556-9984 https://orcid.org/0000-0002-9429-8711 https://orcid.org/0000-0003-0542-2758 https://orcid.org/0000-0002-2791-1037

\section{REFERENCES}

1. Congdon NG, Friedman DS, Lietman T. Important causes of visual impairment in the world today. JAMA 2003;290:2057-60.

2. Colijn JM, Buitendijk GHS, Prokofyeva E, Alves D, Cachulo ML, Khawaja AP, et al. Prevalence of age-related macular degeneration in Europe: the past and the future. Ophthalmology 2017;124:175363.

3. Schaumberg DA, Christen WG, Buring JE, Glynn RJ, Rifai N, Ridker PM. High-sensitivity C-reactive protein, other markers of inflammation, and the incidence of macular degeneration in women. Arch Ophthalmol 2007;125:300-5.

4. Smith BT, Joseph DP, Grand MG. Treatment of neovascular agerelated macular degeneration: past, present and future directions. 
Curr Opin Ophthalmol 2007;18:240-4.

5. Snow KK, Seddon JM. Do age-related macular degeneration and cardiovascular disease share common antecedents? Ophthalmic Epidemiol 1999;6:125-43.

6. van Leeuwen R, Boekhoorn S, Vingerling JR, Witteman JC, Klaver CC, Hofman A, et al. Dietary intake of antioxidants and risk of agerelated macular degeneration. JAMA 2005;294:3101-7.

7. Park SJ, Kwon KE, Choi NK, Park KH, Woo SJ. Prevalence and incidence of exudative age-related macular degeneration in South Korea: a nationwide population-based study. Ophthalmology 2015;122:2063-70.

8. Oesterle A, Laufs U, Liao JK. Pleiotropic effects of statins on the cardiovascular system. Circ Res 2017;120:229-43.

9. Forouzandeh F, Salazar G, Patrushev N, Xiong S, Hilenski L, Fei B, et al. Metformin beyond diabetes: pleiotropic benefits of metformin in attenuation of atherosclerosis. J Am Heart Assoc 2014;3: e001202.

10. Kortekaas KE, Meijer CA, Hinnen JW, Dalman RL, Xu B, Hamming $\mathrm{JF}$, et al. ACE inhibitors potently reduce vascular inflammation, results of an open proof-of-concept study in the abdominal aortic aneurysm. PLoS One 2014;9:e111952.

11. Chrysant SG, Chrysant GS. The pleiotropic effects of angiotensin receptor blockers. J Clin Hypertens (Greenwich) 2006;8:261-8.

12. Ma L, Wang Y, Du J, Wang M, Zhang R, Fu Y. The association between statin use and risk of age-related macular degeneration. Sci Rep 2015;5:18280.

13. Etminan M, Brophy JM, Maberley D. Use of statins and angiotensin converting enzyme inhibitors (ACE-Is) and the risk of age-related macular degeneration: nested case-control study. Curr Drug Saf 2008;3:24-6.

14. Shin JY, Choi NK, Jung SY, Kim YJ, Seong JM, Park BJ. Overlapping medication associated with healthcare switching among Korean elderly diabetic patients. J Korean Med Sci 2011;26:1461-8.

15. Cho GJ. Clinical research using medical big data. Anesth Pain Med 2017;12:9-14.

16. National Health Insurance Service (NHIS). Manual of the National
Health Insurance Service-National Elderly Cohort (NHIS-NEC), South Korea [Internet]. Wonju: NHIS; 2017 [accessed on 2018 March 12]. Available at: https://nhiss.nhis.or.kr/bd/ab/bdaba008cv.do.

17. Suissa S. The Quasi-cohort approach in pharmacoepidemiology: upgrading the nested case-control. Epidemiology 2015;26:242-6.

18. Smeeth L, Cook C, Chakravarthy U, Hubbard R, Fletcher AE. A case control study of age related macular degeneration and use of statins. Br J Ophthalmol 2005;89:1171-5.

19. Klein R, Klein BE, Tomany SC, Danforth LG, Cruickshanks KJ. Relation of statin use to the 5 -year incidence and progression of agerelated maculopathy. Arch Ophthalmol 2003;121:1151-5.

20. Hall NF, Gale CR, Syddall H, Phillips DI, Martyn CN. Risk of macular degeneration in users of statins: cross sectional study. BMJ 2001;323:375-6.

21. McGwin G Jr, Owsley C, Curcio CA, Crain RJ. The association between statin use and age related maculopathy. Br J Ophthalmol 2003;87:1121-5.

22. McCarty CA, Mukesh BN, Guymer RH, Baird PN, Taylor HR. Cholesterol-lowering medications reduce the risk of age-related maculopathy progression. Med J Aust 2001;175:340.

23. McGwin G Jr, Xie A, Owsley C. The use of cholesterol-lowering medications and age-related macular degeneration. Ophthalmology 2005;112:488-94.

24. van Leeuwen R, Tomany SC, Wang JJ, Klein R, Mitchell P, Hofman $\mathrm{A}$, et al. Is medication use associated with the incidence of early age-related maculopathy? Pooled findings from 3 continents. Ophthalmology 2004;111:1169-75.

25. Thomas AS, Redd T, Hwang T. Effect of systemic beta-blockers, ACE inhibitors, and angiotensin receptor blockers on development of choroidal neovascularization in patients with age-related macular degeneration. Retina 2015;35:1964-8.

26. Sedgwick P. Nested case-control studies: advantages and disadvantages. BMJ 2014;348:g1532.

27. Park BJ, Sung JH, Park KD, Seo SW, Kim SW. Report of the evaluation for validity of discharged diagnoses in Korean Health Insurance database. 1st ed. Seoul: Seoul National University; 2003. 